\title{
The effect of a lip bumper on lower dental arch
}

\section{dimensions and tooth positions}

\author{
Johannes Grossen and Bengt Ingervall \\ Orthodontic Clinic, University of Bern, Switzerland
}

SUMMARY The effect of a lip bumper on the dimensions of the lower dental arch and on the inclination of the incisors and first molars was studied in 40 children, aged 9-12 years. The children wore their lip bumper full time for 7-10 months.

The effects of the lip bumper therapy were recorded on dental casts and profile cephalograms made before and after the treatment. The positions and stages of development of the lower second molars were determined on pretreatment intra-oral radiographs.

The lip bumper treatment resulted in an increase of the dental arch widths between the molars, premolars, and canines. The arch length increased through proclination of the incisors and uprighting of the first molars. The stages of development and the positions of the second molars had no influence on the effect of the treatment. Simultaneous treatment in the maxilla with a headgear, a transpalatal arch or a removable plate had no influence on the outcome of the lip bumper therapy.

\section{Introduction}

With the current trend of a non-extraction treatment approach, the use of a lip bumper for gaining arch length has become increasingly popular. The lip bumper is usually worn in the lower jaw and is anchored with buccal tubes to the first molars. The lip bumper lies away from the labial surfaces of the premolars and anterior teeth. These teeth are, therefore, at least partly relieved from the pressure from the cheeks and lip. This changes the conditions of equilibrium. The forces on the lingual surfaces of the teeth, from the tongue, predominate, which leads to proclination of the incisors as reported by amongst others, Bergersen (1972), Bjerregaard et al. (1980), Osborn et al. (1991), Soo and Moore (1991), and Nevant et al. (1991). A significant and clinically important effect of the lip bumper is also the transverse expansion of the dental arch (Cetlin and Ten Hoeve, 1983; Osborn et al., 1991; Nevant et al., 1991).

A lip bumper also has an uprighting effect on the anchorage teeth. The pressure from the lip on the lip bumper gives rise to a force which is transferred to the anchorage teeth (usually the first molars) and may upright and move these teeth distally (Bergersen, 1972; Bjerregaard et al., 1980; Osborn et al., 1991; Nevant et al., 1991).
Lip bumpers may be of varying design, either with a plastic shield in the vestibular fold or simply consisting of a fairly rigid steel wire covered with plastic tubing. The design with a vestibular shield has been shown to have a greater effect on the anchorage teeth (Nevant et al., 1991), probably because the larger contact area with the lip gives rise to greater forces. The wire/plastic tubing design, on the other hand, may be more convenient for the patient and therefore allow full-time use. To have an effect on the dental arch and tooth position, lip bumper therapy usually has to be maintained for periods of up to a year or longer. Treatment with a lip bumper is indicated for space gaining and to reduce anterior crowding by the use of the leeway space. The lip bumper is often used as a simple treatment in the lower jaw simultaneously with the use in the upper jaw of other types of appliances and is ideally suited for interceptive treatment.

Clinical experience with lip bumpers is gratifying and this type of treatment will probably therefore have a definite place in future orthodontics. Further studies of the effect of a lip bumper are needed, however, to clarify such factors as the optimal age of the patient, stage of development and position of the second molars, and the influence of maxillary treatment 
procedures on the outcome of the treatment. This is the aim of the present investigation.

\section{Subjects and methods}

Forty children ( 15 boys and 25 girls) participated in the study. Their ages varied between 8 years, 9 months and 12 years, 4 months (median age 10 years, 3 months). They were treated in the lower jaw with a lip bumper for $7-10$ months (median time 8 months). This was the only appliance used in the lower jaw, but 22 of the children simultaneously used a headgear anchored on the upper first molars, nine had a transpalatal arch, and 10 an upper removable plate. The type of lip bumper used is shown in Fig. 1. It was anchored in buccal tubes on the lower first molars and was adjusted to be positioned $6 \mathrm{~mm}$ below the edges of the lower incisors, and to lie $3 \mathrm{~mm}$ away from the labial surfaces of the incisors and canines, and from the buccal surfaces of the premolars. Control visits were scheduled every month, at which the position of the lip bumper was checked and adjusted if necessary.

The lip bumper was made of 1.1-mm stainless steel wire which was covered with plastic tubing. The children were instructed to wear their lip bumper day and night, and to remove it only for meals or for tooth brushing.

The effects of the lip bumper treatment were documented by measurements on dental casts and profile cephalograms made immediately before and after the lip bumper treatment. The recording on the cast included the measurement of the width of the lower dental arch at the first permanent molars, premolars and canines. The measuring points are shown in Fig. 2. When the premolars or permanent canines were not erupted, the corresponding points on the deciduous teeth were used. No measurement was made when a deciduous tooth was replaced by its successor during the period of observation. The length of the lower dental arch was measured from a line connecting the mesial edge of the buccal tube on the right and left
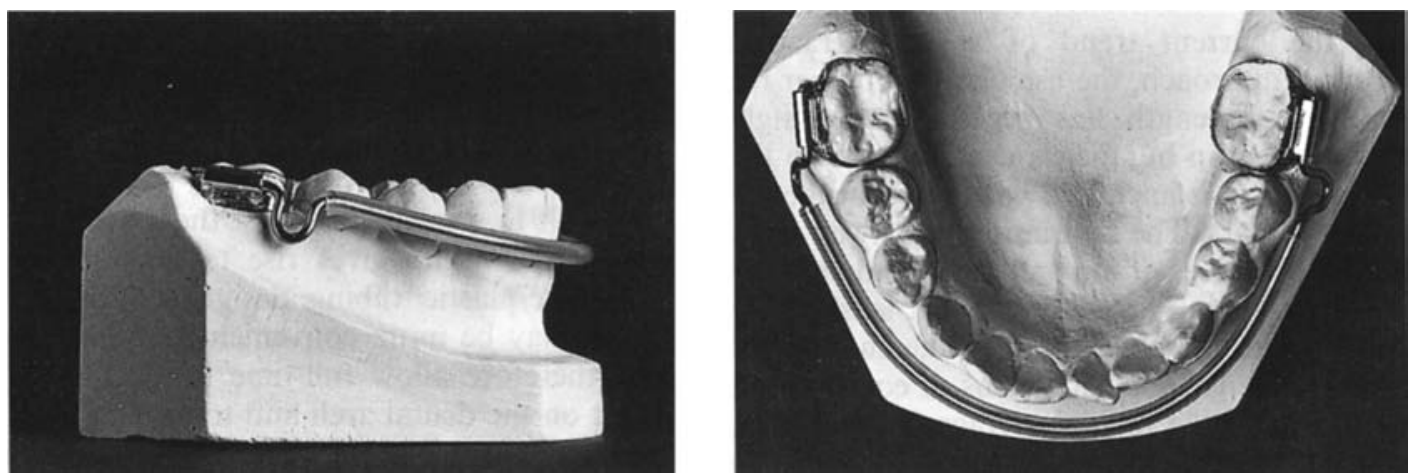

Figure 1 Type of lip bumper used in the study.
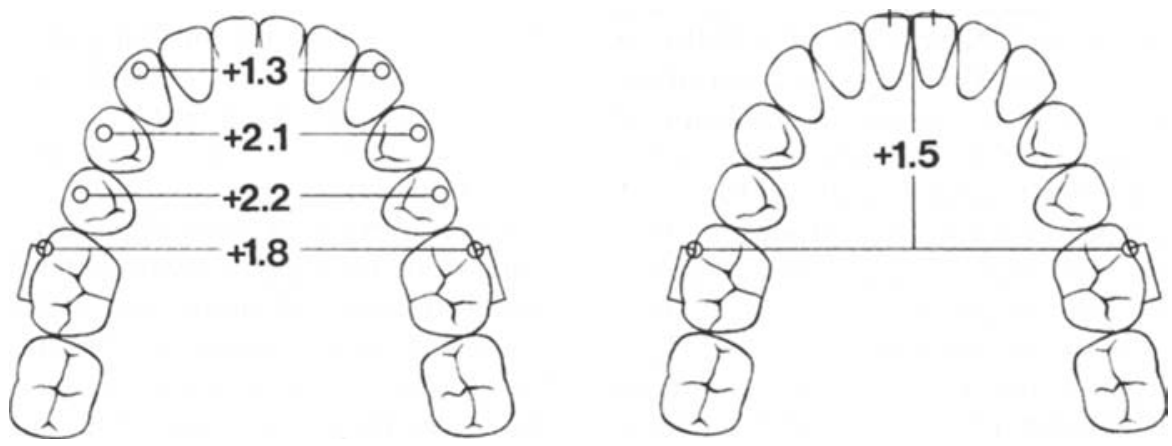

Figure 2 Measuring points used in the recording of the dental arch dimensions. The figure also shows the mean changes in widths and arch length during the period of observation. 
first molars to the midpoint of the incisal edge of the two central incisors. The mean of the measurement to the right and left incisor was used as the variable for arch length. All measurements were made with sliding calipers to the next tenth of a millimeter.

The reference points and lines used in the measurements on the cephalograms are shown in Figure 3. The linear enlargement on the cephalogram was 3.3 per cent (included in the results). The linear measurements on the cephalogram were made as the distance to the line MLP. This line was constructed as a perpendicular to the mandibular line (ML) through pogonion (pg). The mandibular line and $\mathrm{pg}$ were transferred from the cephalogram taken before to the cephalogram taken after treatment by superimposition on the natural reference structures of the mandible (Björk and Skieller, 1983). The linear measurements comprised the distance from MLP to the edge of the lower incisor (ii), to the most prominent part of the labial surface of the lower incisor (il), to the B-point, to the apex of the lower incisor (ia), and to the lower molar $(\mathrm{m})$. The measuring point on the lower molar was located on a metal rod, which before radiography was inserted in the buccal tube on the right lower first molar. The metal rod had a 'flag' extending gingivally. The measuring point was located at the mesial intersection between the rod and the flag (Fig. 3). The inclination of the lower incisor was measured as the angle between $\mathrm{IL}_{\mathrm{i}}$ and $\mathrm{ML}$, and that of the lower molar as the angle between

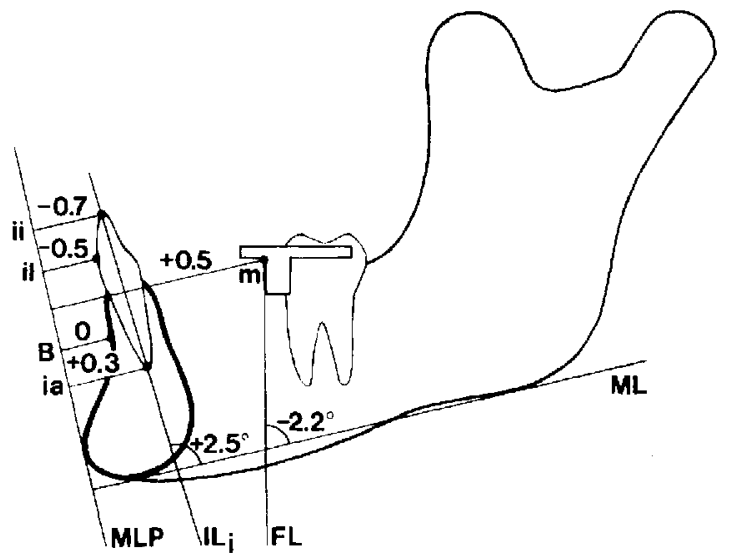

Figure 3 Reference points and lines used in the measurements on the cephalograms. The figure also shows the mean changes during the period of observation.
ML and a line extending to the mesial edge of the flag (FL, Fig. 3). In addition, the overjet and overbite were measured on the cephalograms which were taken in the intercuspal mandibular position. The measurements on the cephalograms were made with the computerbased measuring system of Gebauer (1977).

The positions of the lower second permanent molars before the start of the treatment were evaluated on intra-oral radiographs. The evaluation was made by the two authors using the following grades:

(1) the second molar is covered with bone and is not in contact with the first molar;

(2) close contact between first and second molars;

(3) the second molar is erupted.

The mean of the grading for the right and left sides was used as the variable for second molar position and stage of development.

\section{Statistical methods}

The results presented are the difference between the measurements made on the casts and cephalograms made before and after treatment. The difference was tested for significance with Wilcoxon's matched-pairs, signed-ranks test. Differences between groups were tested with Mann-Whitney's $U$-test. Correlation between variables was calculated with the rankcorrelation of Spearman.

Systematic differences between duplicate determinations were tested with Wilcoxon's matched-pairs, signed-ranks test. The accidental error $\left(s_{i}\right)$ for duplicate determinations was calculated with the formula

$$
s_{i}=\sqrt{\frac{\Sigma d^{2}}{2 n}}
$$

where $d$ is the difference between the two determinations.

\section{Errors of the method}

In order to evaluate the errors of the methods, duplicate measurements (including new superimpositions) were made on 10 randomly selected pairs of casts and cephalograms. No systematic differences were found for the measurements made on the casts. The accidental errors $\left(s_{i}\right)$ for these measurements varied between 0.15 and $0.46 \mathrm{~mm}$. Two of the measurements made on the cephalograms differed systematically 
Table 1 Mean, standard deviation (SD), and range (mm) of changes in the dimensions of the lower dental arch during the treatment. The table also gives the annual changes in matched control groups of the untreated sample of Moyers et al. (1976).

\begin{tabular}{lrllrr}
\hline Width between & $n$ & Mean & SD & Range & $\begin{array}{l}\text { Moyers } \\
\text { et al. }\end{array}$ \\
\hline First molars & 39 & $1.8^{* * *}$ & 2.4 & $-5.0-6.2$ & 0.4 \\
Second premolars & 5 & 2.2 & 2.0 & $-0.4-4.0$ & 0.1 \\
Second deciduous molars & 22 & $1.7^{* * *}$ & 1.3 & $-0.3-4.1$ & 0.0 \\
First premolars & 13 & $2.1^{* *}$ & 1.4 & $0.0-4.1$ & 0.3 \\
First deciduous molars & 12 & $0.7^{* *}$ & 0.7 & $-0.1-2.5$ & -0.1 \\
Canines & 18 & $1.3^{* * *}$ & 1.1 & $-0.5-3.9$ & 0.1 \\
Deciduous canines & 2 & 0.3 & 0.7 & $-0.2-0.8$ & -0.1 \\
Arch length & 39 & $1.5^{* * *}$ & 0.9 & $-0.5-3.6$ & -0.2 \\
\hline
\end{tabular}

** $0.001<P<0.01 ; * * * P<0.001$.

between the duplicate determinations. Thus, the mean change of the distance from MLP to ia was $0.2 \mathrm{~mm}$ and the mean change of the inclination of the lower incisor 1.1 degrees larger at the first than at the second measurement $(0.01<P<0.05$ and $0.001<P<0.01)$. The accidental errors of the linear measurements made on the cephalograms varied between 0.30 and $0.48 \mathrm{~mm}$. The accidental error for the change in inclination of the lower incisor was 1.20 degrees and of the lower molar 1.46 degrees.

\section{Results}

Only one of the variables differed significantly with sex. The mean change of the distance MLP-il was smaller in boys than in girls $(-0.2$ and $-0.6 \mathrm{~mm}$, respectively, $0.01<P<0.05)$. Therefore, the effects of the treatment were analysed for the sexes combined. The changes of the dimensions of the lower dental arch during the treatment are given in Table 1. The table also gives the annual changes in the untreated sample of Moyers et al. (1976). For this comparison, the sample of Moyers et al. was matched with the present individuals with regard to sex and age. This matching was done separately for each variable of Table 1.

As is evident from Table 1, there was a significant increase of the arch length and the widths measured at the first molars, first premolars, and at the permanent canines during the treatment. The widths measured at the deciduous molars also increased significantly. The width at the first molars decreased in five cases. The extreme decrease of $5 \mathrm{~mm}$ as is evident from the range was attributable to mishandling of the lip bumper in one case; the next largest decrease was $2 \mathrm{~mm}$.

The changes during the treatment of the variables recorded on the cephalograms are shown in Table 2.

The lower incisors proclined during the treatment, which resulted in significant decreases of the distances from the vertical reference line (MLP) to the edge and labial surface of the incisor. The lower incisor tipped around a fulcrum located at a point along the root as the apex of the incisor moved in a lingual direction. There was no change in the distance to the B-point but the molar moved significantly posteriorly (distance MLP-m). As is evident from the range, the variation of the changes in inclination of the incisors and molars was considerable.

The effects of the treatment with the lip bumper did not differ between cases treated in

Table 2 Mean, standard deviation (SD) and range of changes during the treatment of variables recorded on the cephalograms $(n=40)$.

\begin{tabular}{lccl}
\hline & Mean & SD & \multicolumn{1}{l}{ Range } \\
\hline Distances (mm) & & & \\
MLP-ii & $-0.7^{* * *}$ & 0.8 & $-3.3-0.3$ \\
MLP-ii & $-0.5^{* * *}$ & 0.6 & $-2.1-0.4$ \\
MLP-B & 0.0 & 0.6 & $-1.8-1.1$ \\
MLP-ia & $0.3^{* *}$ & 0.6 & $-1.2-1.6$ \\
MLP-m & $0.5^{*}$ & 1.1 & $-1.4-3.0$ \\
Overjet & $-1.0^{* * *}$ & 1.6 & $-5.7-1.1$ \\
Overbite & 0.4 & 1.8 & $-3.1-5.4$ \\
Angles (degrees) & & & \\
ILi/ML & $2.5^{* * *}$ & 2.3 & $-1.7-9.3$ \\
FL/ML & $-2.2^{* *}$ & 4.7 & $-11.2-9.1$ \\
\hline
\end{tabular}

${ }^{*} 0.01<P<0.05 ;{ }^{* *} 0.001<P<0.01,{ }^{* * *} P<0.001$. 
the upper arch with a headgear, transpalatal arch, or removable plate, respectively, and cases not receiving such treatment. No significant correlations were found between variables expressing treatment effects and the stage of development of the second molars. The age of the subject was not significantly correlated to the effects of the treatment, with two exceptions. Thus, the width between the first deciduous molars and the distance from MLP to the $\mathrm{B}$-point increased more in older subjects ( $\mathrm{rho}=$ $0.62,0.01<P<0.05$ and tho $=0.39,0.001<$ $P<0.01$, respectively). The change of the dimensions of the dental arch was generally negatively correlated to the value before treatment, i.e. the change tended to be large in subjects with a small dental arch before treatment. None of the coefficients of correlation reached the level for significance, however.

There were negative correlations between the changes in inclination of the lower incisor and molar, respectively, and the inclination before treatment (rho $=-0.29$ and $-0.35,0.01<$ $P<0.05$ ). This means that the incisors tended to procline more during the treatment in cases with relatively upright incisors before treatment. The molar tended to tip distally more in cases where the molar was relatively mesially inclined before treatment.

\section{Discussion}

The effect of the treatment on the lower dental arch dimensions in this study agree closely with the results of earlier studies where the same type of lip bumper was used (Osborn et al, 1991; Nevant et al., 1991). Lip bumper treatment leads to a considerable transverse expansion and increase in arch length which is much greater than from normal growth (Moyers et al., 1976). The increase in arch length was attained through proclination of the incisors and distal tipping of the first molars. This result is in accordance with that of Osborn et al. (1991) who, like us, found a small distal movement of the molars. The changes in incisor and molar inclination in this study and in those of Osborn et al. (1991) and Nevant et al. (1991) are very similar. In the study of Nevant et al. (1991), however, the molar uprighting did not contribute to the increase in arch length because it occurred as a mesial movement of the molar root with the crown remaining stationary.
Another difference from the study of Nevant et al. (1991) is the movement of the lower incisor. In our study, as in that of Bjerregaard et al. (1980), the lower incisor proclination was found to occur as a tipping around a fulcrum within the root. This was evident from the fact that the incisor apex moved in the opposite direction to the crown. In contrast, Nevant et al. (1991) found the apex to remain stationary and consequently the tipping to occur around a fulcrum at the apex.

The measurement from the vertical reference line (MLP) to the B-point was included in the present study in order to reveal if the elimination of the lower lip pressure from the front teeth would lead to apposition of bone at the bone lamella labial to the incisor roots. Such an effect in the region of the B-point has been demonstrated by the use of a Fränkel appliance with its vestibular shield (Fränkel et al., 1987). It was explained by the elimination of a restricting influence from hypertonic circumoral musculature on the dentoalveolar development. In our study, however, no change in the position of the B-point was found. The period of observation in our study was, however, much shorter than in the investigation of Fränkel et al. (1987). The lip bumper is also not comparable to a vestibular shield in protecting the alveolar process from pressure from the musculature.

The effect of a lip bumper did not differ with sex and was unrelated to the age of the patient. These results are in accordance with those of Osborn et al. (1991). In their study, as in our investigation, the lip bumper was used in the mixed or young permanent dentition. Whether the lip bumper will also have an effect in older individuals cannot be determined from our study or from those of Bjerregaard et al. (1980), Osborn et al. (1991), and Nevant et al. (1991), all of whom included only individuals of a similar, young age. In this age-group the stages of development and position of the second molar were not factors affecting the treatment. The stage of development of the second molar was not significantly correlated to any of the variables related to treatment effect. The second molar was scored 2 or 3 , i.e. in close contact with the first molar or erupted, in 12 children. In these children, the mean molar uprighting was 2.9 degrees, i.e. somewhat above the average of the whole sample. The mean distal movement of the molar (variable MLP-m) in these children was $0.6 \mathrm{~mm}$. 
Orthodontic treatment in the maxilla had no influence on the outcome of the lip bumper therapy. It is possible that distal movement of the upper first molars with a headgear would have increased the distal movement of the lower molar by the lip bumper as an effect of occlusal interlocking. In other cases, the unlocking of the occlusion by the wearing of an upper removable plate may be thought to augment the treatment effect of the lip bumper by eliminating hindering occlusal locking. No such effects were found as the changes brought about by the lip bumper were independent of treatment in the maxilla.

It is evident that the amount of space in the dental arch gained by lip bumper treatment can be clinically significant. The combination of an increase in width and length of the dental arch, which is the effect of the lip bumper, is the most effective way of increasing the arch perimeter (Germane et al., 1991). Whether the changes in tooth positions and increases in arch dimensions brought about by a lip bumper are more stable than similar changes achieved in a shorter time with mechanotherapy remains to be studied. It is not known whether the relatively slow expansion of the dental arch by "natural' forces brings about adaptations in surrounding structures which decrease the risk for relapse. That this may be the case is suggested by the results of Soo and Moore (1991), who found the pressure from the lip on the lower front teeth to decrease after lip bumper therapy. A study comparing the effect of lip bumper therapy and mechanical expansion of the dental arch with regard to soft tissue pressure on the teeth after the treatment is being undertaken.

\section{Address for correspondence}

Professor Bengt Ingervall

Klinik für Kieferorthopädie

Freiburgstrasse 7

CH-3010 Bern

Switzerland

\section{References}

Bergersen E O 1972 A cephalometric study of the clinical use of the mandibular labial bumper. American Journal of Orthodontics 61: 578-602

Björk A, Skieller V 1983 Normal and abnormal growth of the mandible. A synthesis of longitudinal cephalometric implant studies over a period of 25 years. European Journal of Orthodontics 5: 1-46

Bjerregaard J, Bundgaard A M, Melsen B 1980 The effect of the mandibular lip bumper and maxillary bite plate on tooth movement, occlusion and space conditions in the lower dental arch. European Journal of Orthodontics 2: $257-265$

Cetlin N A, Ten Hoeve A 1983 Nonextraction treatment. Journal of Clinical Orthodontics 17: 396-413

Fränkel R, Müller M, Falck F 1987 The uprighting effect of the Fränkel appliance on the mandibular canines and premolars during eruption. American Journal of Orthodontics and Dentofacial Orthopedics 92: 109-116

Gebauer U 1977 Elektronische Mess- und Rechenanlage zur arcogrammetrischen Modelldiagnostik und zum Auswerten von Fernröntgenbildern. Schweizerische Monatsschrift Zahnheilkunde 87: 1170-1180

Germane N, Lindauer S J, Rubenstein L K, Revere J H, Isaacson R J 1991 Increase in arch perimeter due to orthodontic expansion. American Journal of Orthodontics and Dentofacial Orthopedics 100: 421-427

Moyers R E, van der Linden F, Riolo M, McNamara J Jr 1976 Standards of human occlusal development. Monograph No 5, Craniofacial Growth Series, Center for Human Growth and Development, University of Michigan, Ann Arbor, Michigan

Nevant C T, Buschang P H, Alexander R G, Steffen J M 1991 Lip bumper therapy for gaining arch length. American Journal of Orthodontics and Dentofacial Orthopedics 100: 330-336

Osborn W S, Nanda R S, Currier G F 1991 Mandibular arch perimeter changes with lip bumper treatment. American Journal of Orthodontics and Dentofacial Orthopedics 99: 527-532

Soo N D, Moore R N 1991 A technique for measurement of intraoral lip pressures with lip bumper therapy. American Journal of Orthodontics and Dentofacial Orthopedics 99: 409-417 